\title{
LONG-TERM VARIABILITY OF
}

\section{EXTRAGALACTIC SOURCES AT 843 MHZ}

\author{
R. W. HUNSTEAD AND B. M. GAENSLER \\ School of Physics, University of Sydney \\ NSW 2006, Australia
}

Time variability is commonly observed in the most compact extragalactic radio sources. Low-frequency variability (LFV) - at frequencies $<1$ $\mathrm{GHz}$ - is thought to arise through two different mechanisms, intrinsic and extrinsic. The former is just an extension of the often rapid high-frequency variations, delayed and reduced in amplitude. The latter is usually attributed to refractive interstellar scintillation (RISS; Rickett et al. 1984), whereby the variations in intensity are the result of wavefront distortions caused by transverse gradients in electron density. If RISS arises predominantly along the signal path through our Galaxy, we might expect to find evidence for a dependence on Galactic coordinates.

Intrinsic variability is most prominent for $\nu>1 \mathrm{GHz}$, while scintillation tends to dominate below $1 \mathrm{GHz}$; variability at $\nu \sim 1 \mathrm{GHz}$ is largely unexplored. The Molonglo Observatory Synthesis Telescope (MOST), operating at $843 \mathrm{MHz}$, is well suited to studying this intermediate regime. Before and after every 12-hour synthesis, MOST records the flux densities of 5-10 compact extragalactic sources, chosen from a list of 55 calibrators (Campbell-Wilson \& Hunstead, 1994). Some 43000 such measurements have been made over the ten year period 1983-1993, forming the database for the present study (Gaensler \& Hunstead, in prep). Some of the MOST 'calibration' sources show quite spectacular variability - see Fig. 1(a).

How do we determine whether a source is varying? The conventional approach is to determine the modulation index $m=\sigma_{S} / S$, where $\sigma_{S}$ is the standard deviation in a source of mean flux density $S$. We first remove unreliable measurements from each dataset to produce a relatively noise-free light curve for each source. To reduce further the random noise contribution to $\sigma_{S}$ we smooth the data by binning into 30-day intervals and then apply a well established test (Fanti et al., 1981) to calculate the $\chi^{2}$ probability that the flux density has remained constant. 

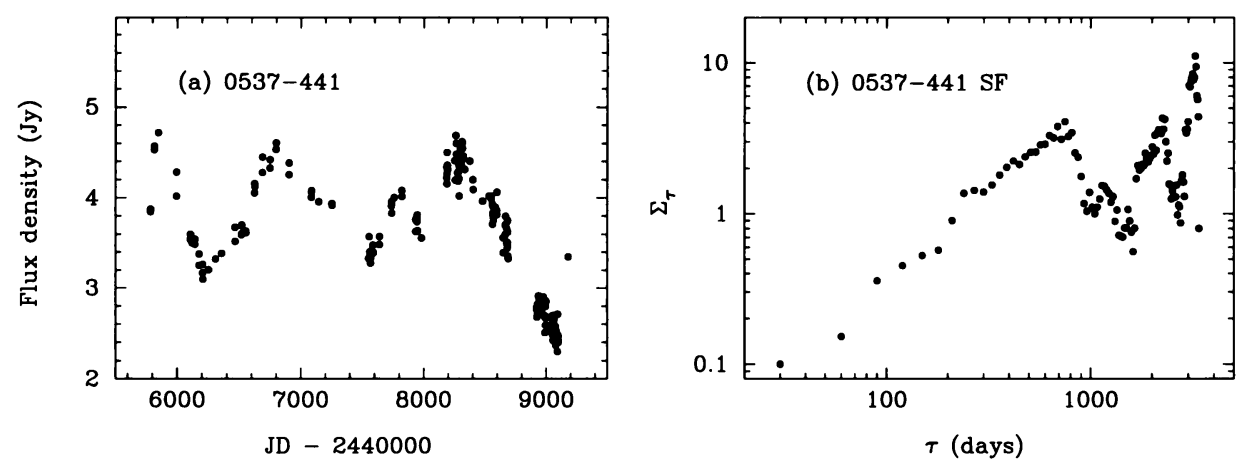

Figure 1. (a) MOST $843 \mathrm{MHz}$ light curve for 0537-441, a peaked-spectrum source identified with a $z=0.894 \mathrm{BL} \mathrm{Lac} / \mathrm{HPQ} / \mathrm{OVV}$ and detected as an X-ray and $\gamma$-ray source; it is core-dominated in the radio, with a diameter of 1.1 mas at $2.3 \mathrm{GHz}$. (b) Structure function for a 30-day smoothed version of the dataset shown in (a).

How do we determine a timescale for the variability? The smoothed dataset is mean-subtracted and normalized by the standard deviation to yield a new time series $\boldsymbol{F}_{t}$. From this we calculate the structure function $\boldsymbol{\Sigma}_{\tau}=\left\langle\left[\boldsymbol{F}_{\boldsymbol{t}+\tau}-\boldsymbol{F}_{t}\right]^{2}\right\rangle$, where $\tau$ is a parameter known as the lag (Spangler et al., 1989). At small lags $\Sigma_{\tau}$ is approximately constant-the noise regimeand as $\tau$ increases $\Sigma_{\tau}$ (when shown on a $\log$-log plot) behaves linearly-the structure regime. Finally there is the saturation regime, where $\Sigma_{\tau}$ flattens out or turns over. We define the timescale $\tau_{V}$ to be twice the lag at which the structure function saturates.

The structure function for 0537-441 in Fig. 1(b) shows a clear linear regime up to $\tau \sim 700$, where it turns over. At greater lags it begins to oscillate about $\Sigma_{\tau} \approx 2$; obviously the longest timescales are poorly sampled. If we interpret the first turnover as saturation this implies $\tau_{V} \sim 1400$ days, which is consistent with the timescales shown in Fig. 1(a).

In all, we find that 17 of the 55 MOST calibrators vary significantly, on timescales ranging from months to years, with 18 non-variables and 20 uncertain. Whereas the non-varying sources are spread uniformly in Galactic latitude, $14 / 17$ variables have $|b|<30^{\circ}$. This clear difference between the two distributions suggests that refractive interstellar scintillation is the dominant cause of LFV at $843 \mathrm{MHz}$.

\section{References}

Campbell-Wilson, D. \& Hunstead, R.W. (1994), PASA, 11, 33.

Fanti, C., Fanti, R., Ficarra, A., Gregorini, L., Mantovani, F., Padrielli, L. \& Weiler, K.W. (1981), A\&AS, 45, 61.

Rickett, B.J., Coles, W.A. \& Bourgois, G. (1984), A\&A, 134, 390.

Spangler, S., Fanti, R., Gregorini, L. \& Padrielli, L. (1989), A \&A, 209, 315. 